\section{HD I01065-a G0 Star with High Metal Content}

ON April 26, 1960, in the course of 2 spectroscopic survey of stars with large proper motions, a spectrum of the eighth magnitude star $H D 101065$ was observed with the Zeiss spectrograph at the Newtonian focus of the 74-in. telescope at Mount Stromlo Observatory. This star, classified as a B5 star in the Henry Draper Catalogue, proved to be a much cooler star with a peculiar spectrum of unknown type. The spectral type of this star as estimated from the continuum, and later confirmed by threecolour photometry undertaken at my request by Mr. J. B. Whiteoak, is K0. The spectrum, however, shows strong hydrogen lines corresponding to class $F 8$ or $G 0$. The assumption that this star may have a composite spectrum could not be substantiated by the appearance of the spectrum in the violet region since the strength of all Balmer lines uniformly corresponds to that of a $G 0$ star. The constant radial velocity of $+2 \cdot 3 \pm 1.0 \mathrm{~km}$. $/ \mathrm{sec}$. (based on three plates) and the six-colour photometry described here also suggest thet the star $H D 101065$ is not a spectroscopic binary.

In view of the fact that no explanation could be found for the discrepancy between the spectral type determined from the continuum and from hydrogen lines (besides the obvious fact that the star in question has a peculiar spectrum) at my request this star was recently observed photoelectrically by Dr. G. E. Kron in six colours (private communication). The results of these observations are shown in Table 1 together with similar data for $H D$ 19373 - a normal main sequence $G 0$ dwarf, $\iota$ Persei ${ }^{1} \ldots$ and $H D 140283$ an extreme $G 2$ sub-dwarf (Stebbins, J., and Kron, G. E., private communication). Like the two other stars, $H D 101065$ is in all probability a dwarf, as the fairly high annual proper motion of $0.054^{\prime \prime}$ and the six-colour photometry suggest. All three stars agree well in three colours, blue, green and red, and there. fore should have essentially the same effective temperature not differing much from that of the Sun. If the difference in the infra-red is interpreted as a temperature effect the star $H D 101065$ should be the hottest and the sub-dwarf $H D 140283$ the coolest, but the difference should not be large. The three stars differ, however, considerably in their violet and ultra-violet colours. The sub-dwarf $H D$ 140283, which according to L. H. Aller and J. L. Greenstein? and $\mathrm{B}$. Baschek ${ }^{3}$ contains only a negligible amount of metals in its atmosphere, possibly only $1 / 200$ of the amount of metals in normal main sequence dwarfs, is the bluest, while the star $H D 101065$ is the reddest. The differences in the violet and ultra-violet are due to the differences in the degree of blanketing, that is, to the differences in the abundance of metals. The star $H D 101065$ should therefore have a much higher abundance of metal in its atmosphere than main sequence dwarfs. This is also suggested by the appearance of its spectrum, which contains about 500 lines, mostly unidentified, in the range $\lambda \lambda 3922-4528 \AA$. on plates with a dispersion of $36 \AA . / \mathrm{mm}$. at $H \gamma$, taken with the three-prism spectrograph at the Cassegrain focus of the 30 -in. Reynolds telescope.

The high abundance of metals is also suggested by the appearance of the continuum. It can be expected that the contribution of metals to the continuous absorption coefficient would suppress the continuum below $4300 \AA$. or possibly up to $\lambda 4400 \AA$., and therefore

\begin{tabular}{|c|c|c|c|c|c|c|}
\hline Colour & $U$ & $V$ & $B$ & $G$ & $R$ & $I$ \\
\hline $\int_{\operatorname{Star}} \lambda \operatorname{in} \AA$ & 3530 & 4220 & 4880 & 5700 & 7190 & 10,300 \\
\hline $\begin{array}{rr}H D & 140283 \\
H D & 19373 \\
H D & 101065\end{array}$ & $\begin{array}{l}-0.52 \\
-0.16 \\
+0.08\end{array}$ & $\begin{array}{l}-0.27 \\
-0.12 \\
+0.06\end{array}$ & $\begin{array}{l}-0.04 \\
=0.03 \\
-0.04\end{array}$ & $\begin{array}{l}-0.01 \\
-0.02 \\
-0.02\end{array}$ & $\begin{array}{l}+0.03 \\
+0.05 \\
+0.07\end{array}$ & $\begin{array}{l}+0.07 \\
+0.13 \\
+0.20\end{array}$ \\
\hline
\end{tabular}

a high content of metals would change the continuum in the sense that the star would appear to be of a later type. Since the spectral type estimated from the continuum and from the three-colour photometry is $K 0$, while the temperature of the star indicates a spectral type G0, the abundance of metals which would cause such a marked change in the appearance of the spectrum must be very high indeed. It is hoped that the spectroscopic study which I have started will provide in the near future a precise figure for the abundance of metals. I expect that the abundance of metals will prove to be many times higher than that of normal main sequence stars.

The energy distribution based on photoelectric spectrophotometry recently secured at my request by Prof. L. H. Aller will be described in another paper now in preparation. This paper will also contain a detailed description of the spectrum; the main features, however, are briefly noted here. The ionized calcium lines $H$ and $K$, which are very prominent in normal $F$ and $G$ dwarfs and even in sub-dwarfs, are faint and approximately equal in strength to hydrogen lines. Also, the absorption spectrum of iron is greatly suppressed and only three lines of iron $(\lambda \lambda 4045,4063,4071 \AA$. .) can be seen beyond any doubt; the line at $\lambda 4045 \AA$. is fairly strong. The lines of ionized strontium at $\lambda 4077 \AA$. and of barium at $\lambda 4554 \AA$. on the contrary are considerably strengthened in comparison with normal dwarfs. The spectrum shows about 500 lines between $\lambda . \lambda .3922$ and $4528 \AA$., the majority of which have not yet been identified. Preliminary identifications seem to suggest that many lines should be attributed to rare earths. The spectrum does not show any $G$ band, which could be interpreted as an indication that the abundance of carbon is low.

The high abundance of barium and strontium-two elements with the magic number of neutrons in their nuclei-as well as the high abundance of rare earths indicate that the star $H D 101065$ is in an advanced stage of stellar evolution. The star apparently passed through a stage of evolution in which heavy elements were built up by slow neutrons. It presents the same evolutionary problems as $S$ stars, complicated in addition by the fact that in all probability it is a dwarf.

I wish to thank Dr. G. E. Kron, who at my request observed the star $H D 101065$ in six colours in spite of the fact that this meant a loss of valuable observing time during his short stay on Mount Stromlo, J. Stebbins and Dr. G. E. Kron for their permission to use unpublished data on $H D$ 140283, and Mr. J. B. Whiteoak for securing the $U B V$ photometry of $H D$ 101065 at a time when this could not be done by me. ANTONI PRZyBYLSKI

Mt. Stromlo Observatory, Australian National University, Canberra.

${ }^{1}$ Stebbins, J., and Kron, G. E., Astrophys. J., 123, 440 (1956).

${ }^{2}$ Aller, L. H., and Greenstein, J. L., Astrophys. J., Supp. No, 46

${ }^{3}$ Baschek, B., Z. Astrophys., 48, 95 (1959). 